\title{
Money bill technology of tactile recognition engravings for visually impaired
}

\author{
A. P. Kyrychok \\ National Technical University of Ukraine "Igor Sikorsky Kyiv Polytechnic Institute", Kyiv, Ukraine \\ Corresponding author. E-mail: akyrychok@ukr.net
}

Paper received 12.02.18; Accepted for publication 22.02.18.

\begin{abstract}
https://doi.org/10.31174/SEND-NT2018-171VI19-07
Abstract. From ancient times man devised systems to give value to things and exchange them, barter was used, and then money arose. Currently, around the world and since 1940, the money has been represented by minted coins and bills issued by each of the Central Banks of the respective countries. The first coins that are known, were coined in Lydia, current Turkey in the seventh century BC, The pioneer in using bills, was the Mongol emperor, Kublai Khan in the eleventh century, for him, was the certificate of property of a quantity of gold coins in Europe, in the beginning, banknotes were certified on the existence of a gold deposit in a bank. The tendency of human beings to exchange things with each other to cover needs seems to be part of the human condition and has been a basic activity in all cultures since the beginning of time known. As the trade spread, people began to travel much more, traveling longer distances, and this fact forced them to travel comfortably and with the least possible weight. The bills represent an easy article to move and to promote the acquisition of products during the journey. But, during those crucial moments of growing commercial exchange for subsistence, nobody publicly explained or figured out how the visually impaired would do to recognize existing money bills. The inalienable need of the visually impaired forced the implementation of adequate ways to identify and differentiate the banknotes by touching the surface of those bills to allow them to obtain the necessary profit of its face value without being victims of unfair merchants.
\end{abstract}

Keywords: banknote design, visualy impered people, producting, money bill, money.

Introduction. According to the figures managed by the World Health Organization, $5 \%$ of the world's population (253 million people) suffer from some type of visual disability, of which 36 million are totally blind, however, and as a result of different inclusion initiatives implemented in most countries of the world, have adapted environments, procedures and regulations to facilitate the lives of people suffering from disabilities of some kind and the visually impaired are among the beneficiaries of these legal, infrastructure adaptations and conformation made and in progress to provide a friendlier world within its limitations and to grant them the necessary protection for their disabilities [Samuel 2010].

The people blind of birth, have a greater capacity of tactile detection than the people with normal vision or partial visual disability, according to a study of the University McMaster in Washington (the United States) published in the magazine The Journal of Neuroscience. The brain records a vision, sound or touch in a split second, the research group, led by Daniel Goldreich, explored whether people with blindness would process the sense of touch more quickly. As Goldreich explains, "our findings reveal that one of the ways in which the brain adapts to the absence of vision is to accelerate the sense of touch." The ability to quickly process non-visual information probably increases the quality of life of the person. People with blindness who rely on an extraordinary degree of non-visual senses" [Wong, Gnanakumaran, \& Goldreich, 2018].

Taking into account, through the empirical knowledge of the developed tactile capacity of the visual impaired from the year 1825 Louis Braille, who was blind from the age of 13 as the result of an accident, perfected a system of 6 points in relief, based on another system of 8-point literacy, previously invented by a French soldier by the name of Charles Barbier de la Serre, to give orders without revealing the positions of the sentinels and taking advantage of the stimuli produced when palpating the reliefs of different types of products. They allowed the visually impaired, to know what product they were deal- ing with at any given moment, being these advances the first steps for the birth of today's Tiflotechnology [Butterly A 2018].

In accordance with all the aspects related to the overdeveloped sense of touch of the vast majority of the visually impaired, including totally blind people, from the year 1966, the De Nederlandsche Bank initiated the development of favorable characteristics for the visually impaired through cleared numbers, and during the year 1971, they were the pioneers with the launching of the first notes with engraved marks for the recognition and differentiation, they varied their size in proportions of $6 \mathrm{~mm}$ by denomination for the visually impaired could easily recognize them, additionally they brought printed large numbers on a light background. The success of these initiatives originated mainly in the fact that they were the first in this successful act because the initiatives arose as a result of the research and work they carried out with a team of 40 blind people that they kept within their workforce [De Heij, 2009, p. 15].

It is also very important to point out that not all the advances made and implemented to facilitate the recognition of banknotes and their denominations, have been well received or accepted at all times by the totally blind people, complaining, in Austria, by the size of the money bill or demanding the non-placement of more dots on them, because these characteristics were assumed as effects of stigmatization towards them, thus they prefered a good general tactility instead, while contrary to their opinions, the National Organization for the visually impaired of Canada congratulated the Bank of Canada for the friendly designs of the banknotes.

The different initiatives studied and implemented with the active participation of groups of the blind and visually impaired in different degrees, by the central banks of each country and the institutions dedicated to the design and manufacture of banknotes of the world, have gone from designs with dots or raised-texture marks, emulating with the points the parameters established with the Braille system, due to the widespread use among the blind and 
the ease of use and recognition. The making of brands for the blind on bills establishes challenges of different types, such as brand type, manufacturing, quality, and durability [Lederman, 2002].

Meanwhile, in other countries of the world, such as the United States, work groups were organized to obtain the best recommendations about the suitable marks for the visually impaired to include them in the new banknotes and to apply those changes to the new designs. Regarding the recommendations, that were related to the size of the money bills according to the denomination, enlarged numbers and with vivid colors. Some concerns emerged from those recommendations, such as the fact that only the difference in size would help the blind while the others features would only help the visually impaired. Additionally, the fact that US dollars banknotes are unchanged and that they are legal tenure and circulation since 1929 until the time of its destruction [Lederman, 2002].

The paper money of united states has no non-visual identification marks, so it is impossible for blind people to determine the value of banknotes without assistance, which results in problems to differentiate the banknotes denominations for a larger population of the visually impaired, including those who, due to aging, lose visual acuity. This detail is mentioned because currently, the authorities in charge of the design, development, and manufacture of the banknotes, are making the necessary efforts to make friendlier banknotes to the users to cover a broader range of citizens who will be facilitated for the recognition of the money bills and denominations.

In the world, there are currently 171 banknote issuers, and some of these entities have addressed the problem incorporating marks for recognition and differentiation, and there are also countries where blind people are provided with portable banknote readers with audio outputs that tell the denomination of the carried banknote [Currency Features for Visually Impaired People 1995].

The manufacture of a banknote is complex, due to the variety of processes that occur from the moment of its design, identifying a very important previous step at the time of the preparation of the base substrate on which the final design will be printed, and it is the impression of watermarks and placement of the security thread according to the corresponding denomination, at the moment of manufacturing the paper money rolls and is carried out by electrolysis processes kept in reserve by the banknote manufacturers. The paper money in most cases is a precise blend of cotton fibers that withstand wear and tear, giving it an approximate duration of 3 to 5 years under normal conditions of use.

Three processes are identified in the manufacture of a banknote: offset printing process; screen printing process; calcography Process (Intaglio Printing).

During the Offset printing process, all background images of the banknote are printed, this printing is done using special permanent fast-drying inks that are very difficult to dilute once dry. Subsequently corresponds the time of screen printing, is made by printing complex images of different colors, which are printed one at a time to complete the final image, in this process the same inks used in the first process mentioned, corresponding, once the screen printing is completed, to the most complex process in the manufacture of banknotes as is the calcographic process. This is the most important of all the manufacturing processes of the banknotes and for its realization, engraved steel sheets are used with all the features that will highlight and give textures to the final banknotes [Kim\&Tuton 2014].

The offset printing process is a smooth printing process, similar to the printing process of any magazine that contains a balanced combination of writing and images, which allows printing in different shades of color in a single pass, during the manufacture of banknotes. It is the first printing process because it is during this that all the images and details that will represent the bottom of the final image of the banknote are printed, it is done simultaneously on both sides of the sheet, and additional security elements are printed as They are: the look security features, hidden numbers, and anti-scan devices. Additionally, the design images are printed in fluorescent ink (invisible image), only observable with ultraviolet light [National research Coouncil 1995].

The screen printing process is a printing technique used in the reproduction of documents and images on any material, and consists of transferring inks through a tensioned mesh, the passage of the ink is blocked in areas where there will be no image, leaving free the area where the ink will pass, it is a repetitive printing system, that once the first model has been achieved, the printing can be repeated hundreds and even thousands of times without losing definition, it is very suitable for printing large simultaneous batches of printed papers, such as series of banknotes.

The intaglio print is a printing technique dating from the fifteenth century used by goldsmiths and reproductions of works of art. from the last century it begins to be used in banknotes and today is one of the pillars in the security of these documents, it is the process used for the generation of a drawing or artistic composition in three dimensions on a plate of engraved metal, these engravings are filled with ink and then transferred to a paper by compressing the metal plate on the paper using a press that exerts a pressure of 20 tons to transfer the ink from the metal plate to the paper, leaving the pattern in high relief .

It is used for the generation of compositions in three dimensions to perform the dry stamping (without ink).

The engraving of the steel plates is done through one of two processes:

1. Hand engraving (engraver): using a cutting tool to remove material from a metal surface.

2. Etching: using acid to remove material from a metal surface.

The application of a great pressure causes a deformation in the surface of the engraved metal sheet, causing that the ink gets transferred to the paper in high relief, being possible to distinguish it to the touch easily, of this process the filaments or threads of ink are detached that in some Tickets are used as an additional security element and they are visible through the use of a magnifying glass. With this system, the main reasons, denomination are stamped and elements such as the latent image, micro prints and tactile marks for the visually and visually impaired are incorporated.

At this point we must mention the modern banknotes manufactured with polymers, which have been in circulation since 1988, the date on which the first units were 
issued by the Australian government as a safe option against counterfeiting, due to their particular characteristics, These bills are manufactured with a biaxially oriented polypropylene polymer (BOPP), which provides excellent qualities in terms of preservation and durability and resistance to weathering and use. The polymer banknotes also incorporate many security measures that are not available on traditional cotton paper tickets, making their counterfeiting much more complicated, likewise incorporating trademarks and bumps for recognition by the visually impaired and the blind. (Butterly, 2018)

The manufacture of the polymer banknotes is done in a similar way to that of the banknotes of cotton fiber in terms of offset, screen printing, and intaglio printing, however there are two specific differentiated, the first is related to the preparation of the base substrate of polymers, which during the process of preparation in sheets, receives two layers of opaque white paint for each of the two surfaces except for this painting the windows that will be intentionally left transparent and a final protective varnishing process of the banknote once its printing process is ready. BOPP banknotes are resistant to folding, tearing, machine and dryer, difficult to burn and recyclable once they reach their useful life date [RBA 2012].

The impression, placement or configuration of recognizable marks by the visually impaired or the blind ranges from the color of the banknote, individual geometric or series marks, groups of dots or individual, numbers enlarged in bright colors to the bumps of the Australian banknotes. This process of mark integration involves a physical or mechanical process that differs when it comes to cotton fiber or polymer banknotes whether it is applied during the printing process or subsequent to it, reminding that the processes vary according to the type of banknote that is being manufactured [Samuel 2010].

When analyzing the printing methodology of recognition marks that facilitate the identification and differentiation of the denominations of the banknotes by the visually impaired or the blind, must be recalled the printing processes to which the banknotes are subjected at the moment of fabrication, specifically to the process of intaglio printing to broaden the explanation of all the subprocesses actively involved in shaping and creating textured marks on the surface of banknotes in specific areas previously chosen through consultation work with visually impaired and blind people, being the best candidates and future users of these features.

The process of intaglio printing begins with the engraving of the marks or sketch on the steel plates that will be used to generate the final drawing or composition in three dimensions, these engravings are filled with ink of the appropriate colors and then transferred to the banknote through the compression of the steel sheet loaded with ink against the paper currency using rollers capable of applying pressures equivalent to 20 tons or more, leaving the drawing in high relief on the surface of the banknote when compressing and embedding the paint on the physical basis of what will be the banknote once finished, in some cases it is required several applications of the necessary pressure due to the degree of complexity of what needs to be recorded as a final finish. In some cases, once the tactile markings are printed, the banknotes are sub- jected to a short baking process at specific temperatures to harden them to their maximum level of resistance.

The quality of details of this process derives from the quality of the engraved marks on the steel sheets, and this quality varies as a consequence of the procedure used to make the engravings, which can be manual or through the use of acid in a process called acid etching.

The first type of engraving involves many hours of dedication by an expert engraver, who works using a series of special tools with which he accurately sizes a previously sketched drawing on the sheet, exchanging tools on measures matters, carving the still surface until finishing the drawing in a detailed way, this type of engraving is slow based on the manual work, when finished a detailed review of each drawing is performed, to determine that all drawings are identical, and once certified, the challenge emerged to print complex banknotes that are identical is solved.

The other engraving process is the chemical engraving, during which the contours corresponding to each drawing are reduced by the successive application of layers of different concentrations of acid, responsible for dissolving the contours of each drawing in a precise and fast manner, reducing the time for obtaining the expected results, currently the engraving procedures of the steel sheets are done in a computerized way by using laser engravers that make detailed drawings, and minimize the needed time to obtain the final etched sheet and to start intaglio printing process.

The treatment applied for the placement of recognition and differentiation marks of type and denomination to paper banknotes is a mechanical treatment, which consist in applying successive layers of ink usually optical variable inks (OVI) on the paper surface by applying high pressure on the two involved surfaces, while the procedures used for Polymer banknotes are physical and are achieved by applying a combination of pressure and focused heat in a selected area of each banknote, and due to the described procedure, create easily discernible bumps, that will be thoroughly used by the visually impaired and the blind to identify any carried or received banknote.

Once the banknotes are printed and bearing the marks of recognition and differentiation that have been chosen to incorporate, there is a qualitative review phase of tests and trials consisting of extensively reviewing all the characteristics, marks dimensions once printed, conformation and look. The banknotes are analyzed by independent groups of people of different specialties invited to participate actively to test the banknotes marks, such as numismatists, notaphily, collectors and similar groups of ordinary citizens made up of chefs, shopkeepers, elders, millennials and youth regardless of race, creed, skin color or diversity. according to the goal to provide all the information about the final finishing of the friendly banknotes for the visually impaired and blind, as the main users of the aforementioned marks.

The most extraordinary and pleasant testing phase is the one that occurs when presenting the bills with incorporated marks, that occurs when a blind person receives the banknote and manages to perceive what for their sense of touch are unmistakable marks that once recognized as such and by the explanation of their meanings to the blind and visually impaired, are incorporated into their spatial 
memory and will indicate to the blind for the rest of their lives the type of banknote and value to which it refers. This is a very important part of the test called the feel test. This test is done to all participants of all groups, however it is the response of the blind people's reaction the most awaited, because through their facial reactions, body language and their expressions of surprise that they convey the real meaning of those marks for their calmness and knowledge about the banknotes from that moment on and further, helping them with their disability.

All the tests are done to all groups of participants, who take note of the opinions and conclusions about each aspect evaluated, to then perform different work dynamics where all of them issue their comments, suggestions, and recommendations, previously stating their criteria about what each one would consider should be the best configuration of marks and which will be the ones with best identification properties. All recommendations are subsequently analyzed and recorded for future improvements of the banknotes to be issued.

Having analyzed all the existing information regarding global initiatives for the inclusion of totally or partially disabled people with the goal of incorporating them into all the life processes enjoyed by any other citizen, it is observed that the technological advances achieved in all areas of life and interaction of human beings without distinction of race, creed, skin color or tendency is a primordial necessity to which solutions must be proposed for the harmonic performance of all humanity.

Having analyzed all the existing information regarding global initiatives for the inclusion of totally or partially disabled people with the goal of incorporating them into all the life processes enjoyed by any other citizen, it is observed that the technological advances achieved in all areas of life and interaction of human beings without distinction of race, creed, skin color or tendency is a primordial necessity to which solutions must be proposed for the harmonic performance of all humanity. The influence of technological advances for the benefit of the visually and visually impaired can be seen in the large number of devices, applications, and banknotes identifiers in the market, however we must be aware that most of the mentioned advances have an implicit cost for the person who wishes to acquire them, while the application of technological advances in the design, and manufacture of banknotes with recognizable engravings by the blind and visually impaired are of domain and public utility.

Conclusion. Nowadays and around the world we witness all the structural modifications and the executed initiatives to include the people that suffer any type of disability, but above all, for those visually impaired or totally blind, presenting as a first change solutions to the problem related to the spatial orientation for the development of multisensory integration and from that development, the blind and visually impaired can travel rooms, streets and cities with relative safety when learning to move and then, with the help of technological advances applied to recognition engravings In banknotes they can use the correct money bills at the right times without being victims of scammers or profiteers.

It is extremely important that all human beings assume the commitment to help others in order to share selflessly with our peers even when they suffer from a disability that may make them perceive as limited, and maintaining the real awareness that in many cases those people with disabilities, in fact, they are more committed than those that are totally healthy.

Always remember blind people perceive their visual impairment as a mere physical challenge.

\section{REFERENCES}

1. Butterly, A. (2018, February 9). How new bank notes will help the visually-impaired community. Retrieved February 9, 2018, from http://www.bbc.co.uk/newsbeat/article/37231241/how-newbank-notes-will-help-the-visually-impaired-community

2. Currency Features for Visually Impaired People (1995). (1995) (1st ed.). National Academy Press.

3. De Heij, H. (2009). DNB Occasional Studies(1st ed., Vol. 7). De Nederlandsche Bank.

4. Kim E and T Turton (2014), 'The Next Generation Banknote Project', RBA Bulletin, March, pp 1-11.

5. Lederman SJ and C Hamilton (2002), 'Using Tactile Features to Help Functionally Blind Individuals Denominate Banknotes', Human Factors, 44(3), pp 413-428.
6. Lederman, S. (2002). Using tactile features to help functionality blind individuals denominate banknotes (1st ed.). US National Library of Medicine National Institutes of Health

7. National Research Council (1995), Currency Features for Visually Impaired People, The National Academies Press, Washington, DC.

8. RBA (Reserve Bank of Australia) (2012), 'Upgrading Australia's Banknotes', Media Release No 2012-27, 27 September.

9. Samuel C (2010), 'Making Banknotes Accessible for Canadians Living with Blindness or Low Vision,' Bank of Canada Review, Winter 2009-2010, pp 29-36.

10. Wong, M., Gnanakumaran, V., \& Goldreich, D. (2018, February 9). Tactile Spatial Acuity Enhancement in Blindness: Evidence for Experience-Dependent Mechanisms. Retrieved February 9, 2018, from http://www.jneurosci.org/content/31/19/7028 